\title{
TITLE:
}

\section{ON THE SIZE OF SOME TRIGONOMETRIC POLYNOMIALS}

$\operatorname{AUTHOR}(\mathrm{S})$ :

Kano, T.

CITATION:

Kano, T.. ON THE SIZE OF SOME TRIGONOMETRIC POLYNOMIALS. 数理 解析研究所講究録 1985, 572: 1-7

ISSUE DATE:

1985-11

URL:

http://hdl.handle.net/2433/99194

RIGHT: 
ON THE SIZE OF SOME TRIGONOMETRIC POLYNOMIALS

T. Kano

Dept. Math., Fac. Sci.

okayama University

Okayama 700 , Japan

Let $E_{n}(f)$ denote the best approximation of $f \in C[0,2 \pi]$, i.e.,

$$
E_{n}(f)=\inf \left\|f-T_{n}\right\|
$$

where $\|$ is the supremum norm and $T_{n}(x)$ are trigonometric polynomials of degree $\leq \mathrm{n}$. It was Bernstein [3] who first showed the close connection between absolute convergence of the Fourier series of $f(x) \in$ Lip $\alpha$ and $E_{n}(f)$. In particular, he obtained the following theorem which shows his result being best possible.

Theorem A. For any given sequence $\varepsilon_{n} \downarrow 0$ such that

$$
\sum_{n=1}^{\infty} \varepsilon_{n} / \sqrt{n}=\infty \text {, }
$$

we can find an $f \in C[0,2 \pi]$ whose Fourier series is not absolutely convergent at any point at all and yet satisfies the inequality $E_{n}(f) \leq \varepsilon_{n}$.

To prove this theorem, he invented the following lemma which may well deserve an independent interest.

Lemma A. For any given natural number $N$, we can find a trigonometric polynomial of the form

$$
\mathrm{T}_{N}(\mathrm{x})=\sum_{\mathrm{N} / 2 \leq \mathrm{n} \leq \mathrm{N}} \cos \left(\mathrm{nx}+\rho_{n}\right)
$$

such that uniformly in $x$,

$$
\mathrm{T}_{N}(\mathrm{x}) \ll \sqrt{\mathrm{N}} .
$$


Bernstein's original proof of this lemma is due to the theory of characters, while Bari [1] applied Kuzmin's lemma instead and Kahane[4]used Rudin-Shapiro theorem. Actually Bernstein considered the sum

$$
S=\sum_{A \leq n \leq B} e\left(a\left(\frac{n^{2}}{N}+x n\right)\right), \quad(e(u)=\exp (2 \pi i u))
$$

where $a>0,0 \leq x \leq I$ and $A, B, N \in N$ are such that $I \leq A<B \leq N$.

First we remark that if we apply Salem's lemma(Lemma 2 below)to $S$, then we obtain

$$
S \ll(\sqrt{a}+1 / \sqrt{a}) \sqrt{N},
$$

which holds uniformly in $x, A, B$.

On the one hand, from a different stand point, there is a problem of finding the polynomials

$$
P_{N}(z)=\sum_{n \leq N} c_{n, N} z^{n}
$$

with $\left|c_{n, N}\right|=1$ and $|z|=1$ such that

$$
\sqrt{\mathrm{N}} \ll \mathrm{P}_{\mathrm{N}}(z) \ll \sqrt{\mathrm{N}},
$$

for all z. Parseval's formula shows

$$
\operatorname{Max}_{|z|=1}\left|P_{N}(z)\right| \geq \sqrt{N} .
$$

See e.g. Kahane[5] for recent results. The next example of (3) in the literature seems to be the following one due to Hardy and Littlewood[cf. 7, p.199]:

$$
P_{N}(z)=\sum_{n \leq N} e(\operatorname{cnlog} n+x n), \quad z=e(x)
$$

which satisfies $\mathrm{P}_{\mathrm{N}}(\mathrm{z}) \ll \sqrt{\mathrm{N}}$ uniformly in $\mathrm{x}$. However, as far as $\mathrm{I}$ know, it seems open whether it satisfies $P_{N}(z) \gg \sqrt{N}$ for all $x$. We notice that in their example the coefficients $c_{n}=e(\operatorname{cnlog} n)$ are independent of $N$.

The main purpose of this note is to show that the size of (3) may be sometimes smaller than $\sqrt{\mathrm{N}}$. We shall show it effectively by constructing examples. 
First we prove

Theorem 1. For any given $N>1$, we can find a sequence $c_{n, N} \in \mathbb{C}$ with $\left|c_{n, N}\right|=1$ ( $\left.1 \leq n \leq N\right)$ such that

$$
\sum_{n \leq N} c_{n, N} z^{n} \ll N^{1 / 4}
$$

for all $z \in \mathbb{C}$ with $|z|=I$, where $\ll$ depends on $z$.

Proof. Consider the sum

$$
S_{N}=\sum_{n \leq N} e(x n-2 s \sqrt{n})
$$

where $0<x \leq 1$ and $s \geq 1$ will be suitably chosen(as a function of $N$ )later. If we put $f(t)=x t-2 s \cdot \sqrt{t}(1 \leq t \leq N)$, then

$$
-s / \sqrt{t}<f^{\prime}(t) \leq 1-s / \sqrt{t} \leq 1-s / \sqrt{N} \text {. }
$$

Therefore, if $4 s^{2} \leq t \leq N$, then

$$
\left|f^{\prime}(t)\right| \leq I-s / \sqrt{N} \text {, }
$$

because then $-(1-s / \sqrt{\mathrm{N}}) \leq-\mathrm{s} / \sqrt{t}$.

Now we shall apply the following known lemma due to van der Corput[cf.2].

Lemma 1. If $f^{\prime}(t)$ is monotone and satisfies

$$
\left|f^{\prime}(t)\right| \leq 1-\varepsilon, \quad(0<\varepsilon<1)
$$

throughout $(a, b)$, then

$$
\sum_{a \leq n \leq b} e(f(n))=\int_{a}^{b} e(f(t)) d t+o(I / \varepsilon),
$$

where the constant implied by 0 is absolute.

If we insert $\varepsilon=s / \sqrt{\mathrm{N}}$ in the above lemma, then we obtain 


$$
4 s^{2} \sum_{\leq n \leq N} e(x n-2 s \sqrt{n})=\int_{4 s^{2}}^{N} e(x t-2 s \sqrt{t}) d t+o(\sqrt{N} / s) .
$$

Thus we have

$$
S_{N}=\sum_{I \leq n \leq 4 s^{2}} e(x n-2 s \sqrt{n})+\int_{4 s^{2}}^{N} e(x t-2 s \sqrt{t}) d t+0(\sqrt{N} / s) .
$$

We appeal to the known lemma below to estimate the first sum in (9).

Lemma 2.(Salem[cf. 7, p.226]). If $f^{\prime \prime}(t)>0$ is monotone, then

$$
\sum_{a \leq n \leq b} e(f(n))=0\left(\operatorname{Max}_{a \leq t \leq b} \frac{1}{\sqrt{f^{\prime \prime}(t)}}\right)+o\left(\int_{a}^{b}\left(\sqrt{f^{\prime \prime}(t)}+f^{\prime \prime}(t)\right) d t\right)
$$

where the implied constants by O's are absolute.

Now for $f(t)=x t-2 s \sqrt{t}$ we have $f^{\prime \prime}(t)=\frac{s}{2} t^{-3 / 2}$. Hence by Lemma 2 we have

$$
\begin{aligned}
& \sum_{1 \leq n \leq 4 s^{2}} e(f(n))=0\left(\operatorname{Max}_{1 \leq t \leq 4 s^{2}} \frac{1}{\sqrt{s}} t^{3 / 4}\right)+O\left(\int_{1}^{4 s^{2}} \sqrt{s} t^{-3 / 4} d t+\right. \\
& \left.\quad+\int_{1}^{4 s^{2}} s t^{-3 / 2} d t\right)=O(s)+o(s)+o(s)=O(s) .
\end{aligned}
$$

Next we shall estinate the integral

$$
I_{N}=\int_{4 s^{2}}^{N} e(x t-2 s \sqrt{t}) d t
$$

If we put $t=u^{2}$, then

$$
\begin{aligned}
I_{N} & =2 \int_{2 s}^{\sqrt{N}} u \cdot e\left(x u^{2}-2 s u\right) d u \\
& =\frac{1}{2 \pi i x} \int_{2 s}^{\sqrt{N}}\left(e\left(x u^{2}-2 s u\right)\right) \cdot d u+\frac{2 s}{x} \int_{2 s}^{\sqrt{N}} e\left(x u^{2}-2 s u\right) d u
\end{aligned}
$$




$$
=\frac{2 s}{x} \int_{2 s}^{\sqrt{N}} e\left(x u^{2}-2 s u\right) d u+0(1 / x) .
$$

Lemma $3[c f .6 \& 7]$. If $f^{\prime \prime}(t) \geq r>0$ throughout ( $\left.a, b\right)$, then

$$
\int_{a}^{b} e(f(t)) d t \ll 1 / \sqrt{r}
$$

where $\ll$ is absolute.

From this lemma we have

$$
\int_{2 s}^{\sqrt{N}} e\left(x u^{2}-2 s u\right) d u=O(1 / \sqrt{x})
$$

Thus we obtain from (9)-(11)

$$
S_{N}=O(s)+O\left(s x^{-3 / 2}\right)+O(\sqrt{N} / s)+O(I / x)
$$

Finally, by choosing. $s=\frac{1}{2} \mathrm{~N}^{1 / 4}$, we get

$$
S_{N}=O\left(N^{1 / 4}\right)
$$

where the implied constant by 0 depends on $x$.

If the coefficients $c_{n, N}$ are independent of $N$, then the situation in general becomes more difficult and we then have the following result.

Theorem 2. For any given $\varepsilon>0$, there exist a natural number $N_{0}=$ $N_{0}(\varepsilon)$ and a sequence $c_{n}=c_{n}(\varepsilon) \in \mathbb{C}$ with $\left|c_{n}\right|=1$ ( $\left.1 \leq n \leq N\right)$ such that for all $N \geq N_{0}$ and $z$ with $|z|=1$,

$$
\sum_{n \leq N} c_{n} z^{n} \underset{\varepsilon, z}{\ll} N^{2 / 5}+\varepsilon
$$

Proof. We only indicate the outline of the proof since it is similar to that of Theorem 1 . In this case we consider the sum 


$$
S_{N}=\sum_{n \leq N} e\left(x n-n^{c} / c\right)
$$

where $0<x \leq I$ and $0<c<I$. If we put $f(t)=x t-t^{c} / c(2 \leq t \leq N)$, then we have by Lemma 1

$$
\sum_{2 \leq n \leq N} e(f(n))=\int_{2}^{N} e(f(t)) d t+O\left(N^{1-c}\right) .
$$

Next we apply a known lemma [7, p.62] in order to estimate the above integral, say $I(N)$. Then after simple calculation, we have for

$$
N \geq 2(2 / x)^{1 /(1-c)}
$$

$$
I(N)-I(N / 2)=O\left(N^{I-3 c / 5}\right)
$$

where $O$ depends on $c$ and $x$. Hence substituting in $N$ successively

$$
\mathrm{N} / 2, \mathrm{~N} / 2^{2}, \ldots \ldots
$$

and adding them all, we get. $I(N)=O\left(N^{1-3 c / 5}\right)$.

Therefore we finally obtain

$$
\begin{aligned}
S_{N} & =O\left(N^{1-3 c / 5}\right)+O\left(N^{1-c}\right)=O\left(N^{1-3 c / 5}\right) \\
& =O\left(N^{2 / 5}+\varepsilon\right), \quad(c=1-5 \varepsilon / 3) .
\end{aligned}
$$

\section{References}

[1] N.K. Bari: A treatise on trigonometric series, vol.2, Pergamon Press, 1964.

[2] E. Beller: Polynomial extremal problems in $\mathrm{L}^{\mathrm{p}}$, Proc. Amer. Math. Soc., 30(1971), 249-259.

[3] S.N. Bernstein: Sur la convergence absolue des séries trigonométriques, Comptes Rendus, Paris, 158(1914), 1661-1664.

[4] J.-P. Kahane: Séries de Fourier absolument convergentes, Springer 1970 .

[5] J.-P. Kahane: Sur les polynomes à coefficients unimodulaires, Bull. London Math. Soc.,12(1980), 321-342. 
[6] E.C. Titchmarsh: The theory of the Riemann zeta-function, Oxford, 1951.

[7] A. Zygmund: Trigonometric series, vol.1, Cambridge, 1959. 